\title{
Hierarchical Modulation with Vector Rotation for E-MBMS Transmission in LTE Systems
}

\author{
Hui Zhao, Xiaoping Zhou, Yunchuan Yang, and Wenbo Wang \\ Key Laboratory of Universal Wireless Communication, Beijing University of Posts and Telecommunications, Ministry of Education, \\ Xitucheng Road no.10, Haidian District, Beijing 100029, China \\ Correspondence should be addressed to Hui Zhao, hzhao@bupt.edu.cn
}

Received 1 April 2010; Revised 4 July 2010; Accepted 6 August 2010

Academic Editor: Raymond Kwan

Copyright ( $\odot 2010$ Hui Zhao et al. This is an open access article distributed under the Creative Commons Attribution License, which permits unrestricted use, distribution, and reproduction in any medium, provided the original work is properly cited.

Enhanced Multimedia Broadcast and Multicast Service (E-MBMS) is considered of key importance for the proliferation of LongTerm Evolution (LTE) network in mobile market. Hierarchical modulation (HM), which involves a "base-layer" (BL) and an "enhancement-layer" (EL) bit streams, is a simple technique for achieving tradeoff between service quality and radio coverage. Therefore, it is appealing for MBMS. Generally, HM suffers from the severe performance degradation of the less protected EL stream. In this paper, HM with vector rotation operation introduced to EL stream is proposed, in order to improve EL's performance. With the proper interleaving in frequency domain, this operation can exploit the inherent diversity gain from the multipath channel. In this way, HM with vector rotation can effectively enhance multimedia broadcasting on quality video and coverage. The simulation results with scalable video coding (SVC) as source show the significant benefits in comparison with the conventional HM and alternative schemes.

\section{Introduction}

Video broadcast and multicast are expected to constitute a significant portion of the load on future mobile communication systems. MBMS and E-MBMS of 3GPP provide the means of delivering mobile TV to the mass market, which has already been standardized in 3GPP UTRAN (UMTS Terrestrial Radio Access Network) Release6,7 , and 8 (R8 is just LTE). But it is still open in R10 (i.e., LTE-Advanced). In this paper, we will discuss the radio transmission of E-MBMS with point-to-multipoint connections. Regarding the feature of multimedia service, the source often adopts the multiple resolution code [1]. Hence, unequal error protection (UEP) transmission is a natural support for this kind of information [2]. Namely, the coarse resolution gets a better protection than the fine resolution. Therefore, the coarse resolution information will be recovered by all receivers to obtain the crude reconstruction while the fine resolution information can only be recovered by some receivers due to propagation conditions. In short, the combination of multiple resolution source code and UEP transmission can balance tradeoff between video quality and robust coverage in media broadcast applications.

As a specific example of UEP transmission, HM is widely used in digital broadcasting systems, such as, DVB-T, MediaFLO, and UMB-BCMCS. HM consists of non-uniform signal constellations to provide unequal bit error protection for $\mathrm{BL}$ and EL streams. Many researches have been carried out on this topic. Some literatures discussed the application of HM in multiple input multiple output (MIMO) systems $[3,4]$ or in LTE system based on Orthogonal Frequency Division Multiplexing (OFDM) [5, 6], while some others reported the joint optimization between HM and separate/joint sourcechannel coding in $[7,8]$. Providing much attention to HM itself, [9] has proposed an enhanced HM scheme along with three optimization criteria, in which the signal constellation of EL stream is rotated by a certain angle, named as "HM with CR" in this paper. The authors of [10] presented a concatenated encoding scheme with HM to well protect the EL stream, which greatly complicates the transceiver structure. 


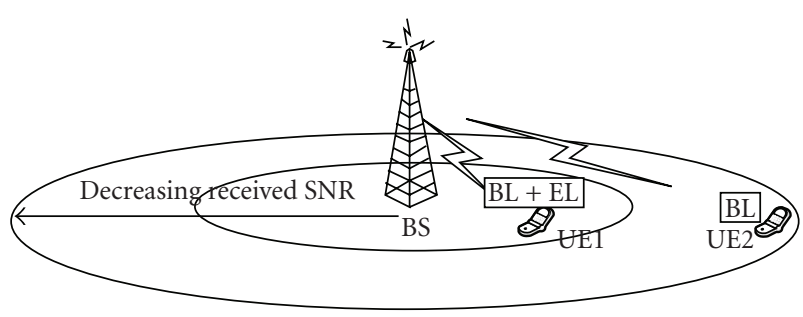

FIgure 1: A broadcast model with HM.

This paper also aims to improve the protection of the EL stream through a simple approach. As we all know, to efficiently decrease the bit-error probability (BER) further, we can resort to exploit the diversity gain under high received signal to noise ratio (SNR) condition or the coding gain in low SNR region. And the user at the center of cell who can recover the EL signal generally has a higher SNR than that at the edge, as illustrated in Figure 1. Hence, this paper applies the idea of signal space diversity (SSD) [11] to provide diversity gain to the EL stream, which is termed as "HM with vector rotation (VR)". Rough predictions on video quality and coverage are given using SVC video as media example. The simulation results in LTE E-MBMS system show that our scheme is better than the existing HM and "HM with CR" due to the diversity gain at the cost of a slight increase in detection complexity.

The rest of the paper is organized as follows: Section 2 introduces the system model of an OFDM system with $\mathrm{HM}$, reviews the definition of "HM with CR", and presents the existing problem. In Section 3, we propose "HM with VR". After the scheme description of transceiver, we give the analysis of diversity gain of EL stream and receiver complexity, comparing with the classical HM. Subsequently, the simulation in Sections 4 and 5 verifies the benefits of our proposed scheme in the video quality and the radio coverage, respectively. Finally, conclusions are drawn in Section 6.

\section{System Model and Problem Description}

Compared to the conventional modulation methods (such as, QPSK, 16-QAM, and 64-QAM, etc.), HM allows the multiplexing transmission of multilayer streams in superposition with different transmission qualities, which stem from the concept of superposition coding in [12]. Without loss of generality, only the two-layer transmission is considered in this paper, that is, one BL and one EL.

2.1. System Model with HM. In Figure 2, we show a transceiver diagram that incorporates HM into a UTRA LTE-based OFDM transmission. Each stream is encoded and mapped to the constellation symbols according to the importance. That is to say, BL stream is mapped to the most significant bits (MSB) and EL to the less significant bits (LSB). After the serial/parallel (S/P) operation, the symbols are then converted to the time domain using Inverse Fast Fourier Transform (IFFT). With the added cyclic prex (CP), the OFDM symbols are sent over the frequency-selective multipath fading channel. The receiver removes the $\mathrm{CP}$ performs FFT and P/S operation. Then the received signal at the $k$ th subcarrier in frequency-domain can be written as

$$
y_{k}=h_{k} x_{k}+n_{k},
$$

where $h_{k}$ is the channel fading coefficient in frequency domain, $x_{k}$ is the complex hierarchical symbol, $n_{k}$ is assumed to be zero-mean circularly symmetric complex Gaussian noise with distribution $n_{k} \sim N_{c}\left(0, \sigma_{n}^{2}\right)$. We only discuss this system model in frequency domain, thus the subscript $k$ can be omitted for short later.

According to the definition of HM, the HM symbol $x$ can be rewritten as

$$
x=\alpha x^{\mathrm{bl}}+\beta x^{\mathrm{el}},
$$

where $x^{\mathrm{bl}}$ and $x^{\mathrm{el}}$ are the symbols, respectively, mapped to MSB and LSB; $\alpha$ and $\beta$ are the power parameters for BL and EL and satisfy $\alpha>\beta$; hence, the power ratio between the two layers is defined as

$$
\eta=\frac{\alpha^{2}}{\left(\alpha^{2}+\beta^{2}\right)} .
$$

This ratio will determine how much more the BL stream is protected against errors than is the EL stream.

The receiver is assumed to have the perfect channel state information. After channel equalization and soft demodulation, the two streams with soft information are sent to the separate decoders.

2.2. HM with $C R$. The regular HM suffers from interlayer interference. In order to recover the capacity loss due to this interference, [9] has presented an enhanced "HM with CR" from an information-theoretical perspective. Corresponding with the regular QPSK/16 QAM HM in Figure 3(a), the principle of "HM with CR" is shown in Figure $3(\mathrm{~b})$. The symbol of EL is rotated in counter-clockwise by $\theta(0 \leqslant \theta \leqslant$ $\pi / 4)$. The resulted symbol is expressed as

$$
x=\alpha x^{\mathrm{bl}}+\beta e^{j \theta} x^{\mathrm{el}} .
$$

The optimal $\theta$ is chosen to maximize the minimum Euclidean distance (MED) of the resulted constellation points, which will lead to a better performance.

2.3. Problem Description. As shown in Figure 1, in a broadcasting system with HM, the users at the edge of cell (e.g., UE2) experience the low SNR channel due to the long distance to the base station (BS). For guaranteeing the recover of the $\mathrm{BL}$ information at the edge, the power allocated to BL should be large enough, while the power allocated to EL must be cut down with the constant transmit power constraint. However, if the EL stream can be well protected also, the users at the center (e.g., UE1) will enjoy the higher video quality. In other words, with the same video quality, the coverage area will be enlarged. As we all know, regarding to the BER performance, the diversity gain becomes critical when SNR is high and the coding gain when 


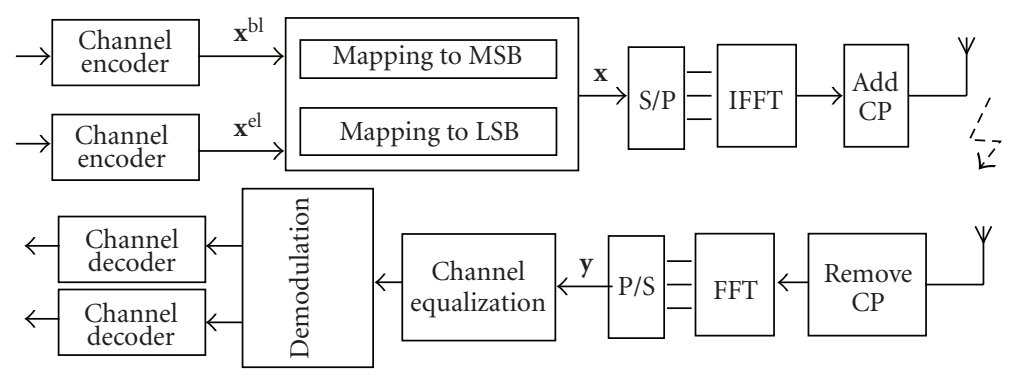

FIgURE 2: Transceiver diagram of OFDM system with HM.

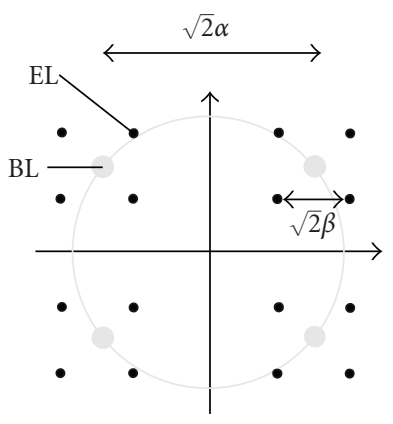

(a)

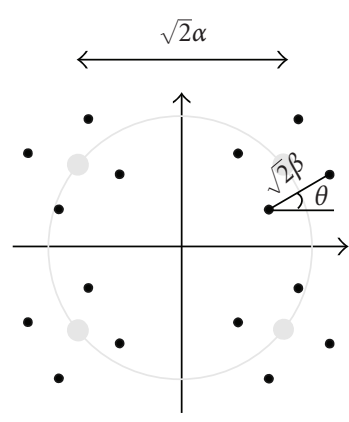

(b)
FIGURE 3: QPSK/16 QAM HM constellation for (a) regular HM (b) "HM with CR".

SNR is in medium or low region. If we neglect the influence of interlayer interference, it is conjectured that the SNR of UE1's EL signal is higher than that of UE2's BL signal in the most cases. Accordingly, we will exploit the diversity gain for EL while $\mathrm{BL}$ has earned the coding gain due to the large allocated power.

\section{Proposed HM with Vector Rotation}

SSD, also named modulation diversity, is a technique to provide the diversity gain for a single antenna system in the fast fading channel. To simply explain, SSD is just used to multiply an $N$-by- $N$ precoding matrix to a group of $N$ modulated symbols (i.e., vector rotation), then let the precoded symbols experience the fully interleaved fading channel. The proper choice of precoding matrix can earn full diversity of $N$ for the symbols. In this paper, we apply SSD in HM for improving the EL performance.

3.1. Scheme Description. Figure 4 illustrates the transceiver diagram of the proposed "HM with VR". The difference between Figures 4 and 2 lies in the following. First, the $N$ continuous EL data symbols mapped to LSB are precoded by a rotation matrix $\mathbf{R}$; second, a pseudorandom symbol interleaving in frequency domain is implemented and the interleaving duration is limited in the range of a codeword; finally, the two streams are separately detected. Here are the details.
Analogous to (2) and (4), the $N$ hierarchical symbols $x_{i}(i=1,2, \ldots, N)$ after the operation of "HM with VR" compose a hierarchical vector as

$$
\mathbf{x}=\left[x_{1}, x_{2}, \ldots, x_{N}\right]^{T}=\alpha \mathbf{x}^{\mathrm{bl}}+\beta \mathbf{R} \mathbf{x}^{\mathrm{el}},
$$

where $\mathbf{x}^{\mathrm{bl}}=\left[x_{1}^{\mathrm{bl}}, x_{2}^{\mathrm{bl}}, \ldots, x_{N}^{\mathrm{bl}}\right]^{T}$ and $\mathbf{x}^{\mathrm{el}}=\left[x_{1}^{\mathrm{el}}, x_{2}^{\mathrm{el}}, \ldots, x_{N}^{\mathrm{el}}\right]^{T}$ are the BL and EL vectors, respectively; $\mathbf{R}$ is an $N$-by- $N$ unitary matrix, referred to [11]. Then in the $N$ continuous symbol durations, the equivalent system model in the frequency domain is

$$
\mathbf{y}=\mathbf{H x}+\mathbf{n},
$$

where $\mathbf{y}=\left[y_{1}, y_{2}, \ldots, y_{N}\right]^{T}$ is the received vector; $\mathbf{H}=$ $\operatorname{diag}\left(h_{1} \quad h_{2} \quad \cdots \quad h_{N}\right)$ is the equivalent channel matrix, whose elements can be regarded as mutually independent due to the assumption of ideal symbol interleaving in frequency domain; $\mathbf{n}=\left[n_{1}, n_{2}, \ldots, n_{N}\right]^{T}$ is the noise vector with zero mean and correlation matrix $\sigma_{n}^{2} \mathbf{I}_{N}$; here $\mathbf{I}_{N}$ denotes an $N$-by- $N$ identity matrix.

Regarding demodulation, the bits of both $\mathrm{BL}$ and $\mathrm{EL}$ streams are jointly judged in HM. But as to "HM with VR", we apply the separate detection to each stream and the interference cancellation (IC) for the sake of complexity simplification. First, the BL data is detected. Here the interference from EL is regarded as a part of the general noise $\mathbf{n}^{\prime}$ :

$$
\widehat{\mathbf{x}}^{\mathrm{bl}}=\alpha^{-1} \mathbf{H}^{-1} \mathbf{y}=\mathbf{x}^{\mathrm{bl}}+\mathbf{n}^{\prime}
$$

where $\mathbf{H}^{-1}$ is the inverse of $\mathbf{H}$.

Then the interference on EL from BL is regenerated and cancelled from the received vector. The resulting received vector of the single EL is

$$
\mathbf{y}^{\mathrm{el}}=\mathbf{y}-\alpha \mathbf{H} \cdot \mathfrak{Z}\left(\hat{\mathbf{x}}^{\mathrm{bl}}\right)=\beta \mathbf{H R} \mathbf{x}^{\mathrm{el}}+\mathbf{n}^{\prime \prime},
$$

where $\mathcal{Z}\left(\hat{\mathbf{x}}^{\mathrm{bl}}\right)$ means demodulating each element of $\hat{\mathbf{x}}^{\mathrm{bl}}$ to the nearest constellation symbol; let $\Delta \mathbf{x}^{\mathrm{bl}}=\mathfrak{Z}\left(\hat{\mathbf{x}}^{\mathrm{bl}}\right)-\mathbf{x}^{\mathrm{bl}}$, which denotes the demodulation error, then $\mathbf{n}^{\prime \prime}=\alpha \mathbf{H}$. $\Delta \mathbf{x}^{\mathrm{bl}}+\mathbf{n}$ is zero mean and correlation matrix $\sigma_{n^{\prime \prime}}^{2} \mathbf{I}_{N}=$ $\alpha^{2} \operatorname{diag}\left(\left|h_{1} \cdot \Delta \mathbf{x}_{1}^{\mathrm{bl}}\right|^{2},\left|h_{2} \cdot \Delta \mathbf{x}_{2}^{\mathrm{bl}}\right|^{2}, \ldots\left|h_{N} \cdot \Delta \mathbf{x}_{N}^{\mathrm{bl}}\right|^{2}\right)+\sigma_{n}^{2} \mathbf{I}_{N}$.

Finally, implement $N$-symbol maximum likelihood (ML) or other near-ML low-complexity detection to $\mathrm{y}^{\mathrm{el}}$ to get the EL data estimation $\hat{\mathbf{x}}^{\mathrm{el}}$. 


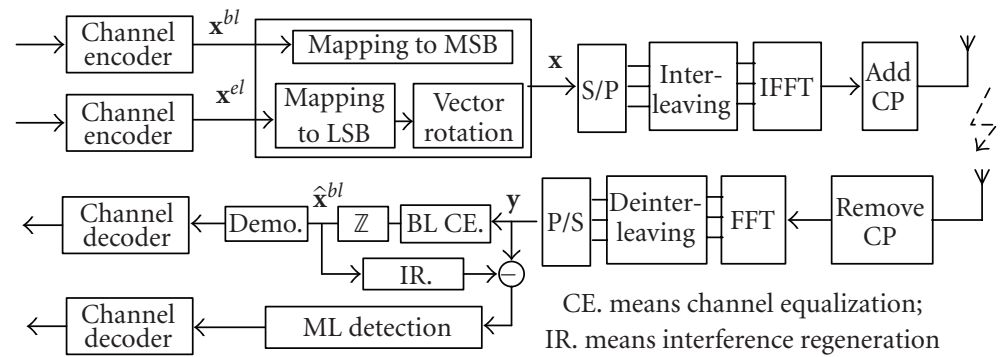

FIgURE 4: Transceiver diagram of the proposed "HM with VR".

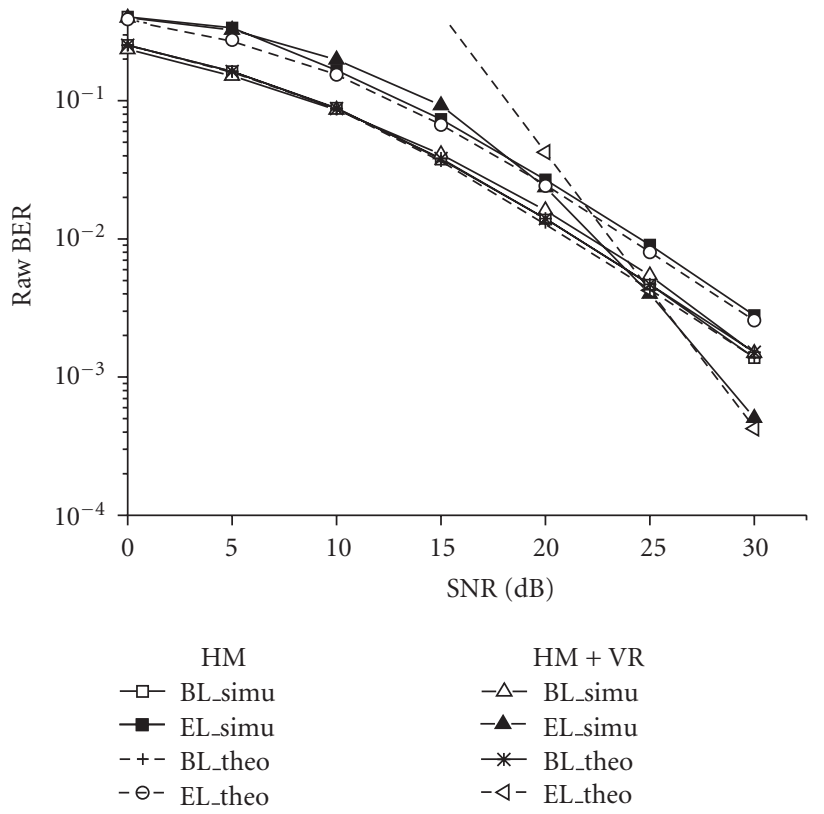

FIGURE 5: Validation of Raw BER formula.

3.2. Analysis on Error Probability. It has been demonstrated in [11] that the symbol diversity order of SSD is equal to the dimension of square rotation matrix $N$ when rotation matrix is designed reasonably and the $N$ rotated symbols are detected jointly. Although the larger $N$ is leading to the more diversity gain, the value of $N$ cannot be too large in this paper, because the performance of $\mathrm{BL}$ will deteriorate due to the interference from EL when $N$ is large. Furthermore, with the consideration of detection complexity, we take $N=2$ in this paper. Here, we give the performance comparison between "HM with VR" and the classical HM by the derivation of bit error probability (BER) for both $\mathrm{BL}$ and EL streams.

In [13], the author has deduced the BER expressions of the generalized hierarchical-QAM constellations over fading channel. Hence, the BER formulas of each stream of HM and BL stream of "HM with VR" can be obtained directly with reference to [13]. As to "HM with CR", the BER expression of EL stream is hard to derive due to the complex decision region. Hence, we only give the simulation comparison in Figure 5.
Before deducing the BER of EL stream of "HM with VR", we give its symbol pairwise error probability (SPEP) firstly to analyze the diversity order. Here we use moment generation function- (MGF-) based approach, different with the canonical probability density function- (PDF-) based approach used in [11].

(1) SPEP. Equation (8) can be rewritten as (the value of $\mathbf{R}$ is from [11])

$$
\mathbf{y}^{\mathrm{el}}=\beta \mathbf{H R} \mathbf{x}^{\mathrm{el}}+\mathbf{n}^{\prime \prime}=\underbrace{\beta\left[\begin{array}{cc}
h_{1} & 0 \\
0 & h_{2}
\end{array}\right]\left[\begin{array}{cc}
\cos \varphi & \sin \varphi \\
-\sin \varphi & \cos \varphi
\end{array}\right]}_{\widetilde{\mathbf{H}}} \mathbf{x}^{\mathrm{el}}+\mathbf{n}^{\prime \prime},
$$

where $\phi=0.5 \arctan (2) ; \tilde{\mathbf{H}}$ is the equivalent channel matrix; here, we observe the diversity order at high SNR so that the error propagation from BL can be neglected and $\mathbf{n}^{\prime \prime}$ has the same distribution as $\mathbf{n}$.

Let the difference vector $\Delta \mathbf{x}^{\mathrm{el}}=\mathbf{x}^{\mathrm{el}}-\widehat{\mathbf{x}}^{\mathrm{el}}=\left[\begin{array}{ll}\delta_{1} & \delta_{2}\end{array}\right]^{T}$; the conditional SPEP for $\mathbf{x}^{\mathrm{el}}$ can be expressed by the Gaussian Q-function as [14]

$$
P\left(\mathbf{x}^{\mathrm{el}} \neq \hat{\mathbf{x}}^{\mathrm{el}} \mid \mathbf{H}\right)=Q\left(\sqrt{\frac{\left|\mathbf{H R} \cdot \Delta \mathbf{x}^{\mathrm{el}}\right|^{2}}{2 \sigma_{n}^{2} / \beta^{2}}}\right)=Q\left(\sqrt{\frac{\mu}{2 \sigma_{n}^{2} / \beta^{2}}}\right),
$$

where $\mu \triangleq\left|\mathbf{H R} \Delta \mathbf{x}^{\mathrm{el}}\right|^{2}=\left(\mathbf{R} \cdot \Delta \mathbf{x}^{\mathrm{el}}\right)^{H} \mathbf{H}^{H} \mathbf{H R} \cdot \Delta \mathbf{x}^{\mathrm{el}}$. According to the definition, one has

$$
\mu=\left|h_{1}\right|^{2} \omega_{1}^{2}+\left|h_{2}\right|^{2} \omega_{2}^{2},
$$

where $\omega_{1}=\left|\delta_{1} \cos \varphi+\delta_{2} \sin \varphi\right|$ and $\omega_{2}=\left|\delta_{1} \sin \varphi-\delta_{2} \cos \varphi\right|$ are real.

We now apply the Craig's formula [15] to derive the conditional SPEP in (10):

$$
\begin{aligned}
P\left(\mathbf{x}^{\mathrm{el}} \neq \hat{\mathbf{x}}^{\mathrm{el}} \mid \mathbf{H}\right) & =Q\left(\sqrt{\frac{\mu}{2 \sigma_{n}^{2} / \beta^{2}}}\right) \\
& =\frac{1}{\pi} \int_{0}^{\pi / 2} \exp \left(\frac{-\mu}{4 \sin ^{2} \theta \cdot \sigma_{n}^{2} / \beta^{2}}\right) d \theta \\
& =\frac{1}{\pi} \int_{0}^{\pi / 2} \exp \left(-\frac{\left|h_{1}\right|^{2} \omega_{1}^{2}+\left|h_{2}\right|^{2} \omega_{2}^{2}}{4 \sin ^{2} \theta \cdot \sigma_{n}^{2} / \beta^{2}}\right) d \theta .
\end{aligned}
$$


Since $h_{1}$ and $h_{2}$ are i.i.d $\sim \mathrm{N}_{c}(0,1)$, we apply MGF-based approach [14] to obtain the unconditional SPEP as follows:

$$
P\left(\mathbf{x}^{\mathrm{el}} \neq \widehat{\mathbf{x}}^{\mathrm{el}}\right)=\frac{1}{\pi} \int_{0}^{\pi / 2}\left[\left(1+\frac{\beta^{2} \omega_{1}^{2}}{4 \sigma_{n}^{2} \sin ^{2} \theta}\right)\left(1+\frac{\beta^{2} \omega_{2}^{2}}{4 \sigma_{n}^{2} \sin ^{2} \theta}\right)\right]^{-1} d \theta .
$$

At high SNR, the upper bound of (13) is given by

$$
\begin{aligned}
P\left(\mathbf{x}^{\mathrm{el}} \neq \hat{\mathbf{x}}^{\mathrm{el}}\right) & \leq \frac{1}{\pi} \int_{0}^{\pi / 2}\left[\left(\frac{\beta^{2} \omega_{1}^{2}}{4 \sigma_{n}^{2} \sin ^{2} \theta}\right)\left(\frac{\beta^{2} \omega_{2}^{2}}{4 \sigma_{n}^{2} \sin ^{2} \theta}\right)\right]^{-1} d \theta \\
& =\frac{1}{\pi}\left[\frac{\beta^{2} \omega_{1}^{2}}{4 \sigma_{n}^{2}} \cdot \frac{\beta^{2} \omega_{2}^{2}}{4 \sigma_{n}^{2}}\right]^{-1} \int_{0}^{\pi / 2} \sin ^{4} \theta d \theta \\
& =\frac{12}{\beta^{4} \omega_{1}^{2} \omega_{2}^{2}}\left(\frac{2}{\sigma_{n}^{2}}\right)^{-2} .
\end{aligned}
$$

Because the transmission power is 2 when $N=2,\left(2 / \sigma_{n}^{2}\right)$ in (14) is just SNR. From (14), we could easily find that the exponent of SNR is -2 , that is, diversity order is two. But for HM, the hierarchical modulated symbols only experience one subcarrier channel so as to only earn the symbol diversity of 1 .

Further, we can find the union bound on the average SPEP, denoted by $\mathrm{PEP}^{\mathrm{el}}$, by traversing all the possible vectors, the size of which is $L$

$$
\mathrm{PEP}^{\mathrm{el}}=\frac{2}{L} \sum_{i=1}^{L-1} \sum_{j=i+1}^{L} P\left(\mathbf{x}_{i}^{\mathrm{el}} \neq \mathbf{x}_{j}^{\mathrm{el}}\right) .
$$

(2) BER. The two symbols of EL are detected jointly, so the error probability of the $i$ th symbol $x_{i}^{\mathrm{el}}$ is related to the other (denoted as $x_{\bar{i}}^{\mathrm{el}}, i, \bar{i} \in[1,2]$ ), which can be expressed as

$$
\begin{aligned}
P\left(x_{i}^{\mathrm{el}} \neq \hat{x}_{i}^{\mathrm{el}}\right)= & \underbrace{P\left(x_{i}^{\mathrm{el}} \neq \hat{x}_{i}^{\mathrm{el}} \mid x_{\bar{i}}^{\mathrm{el}}=\hat{x}_{\bar{i}}^{\mathrm{el}}\right)}_{A}\left[1-P\left(x_{\bar{i}}^{\mathrm{el}} \neq \hat{x}_{\bar{i}}^{\mathrm{el}}\right)\right] \\
& +\underbrace{P\left(x_{i}^{\mathrm{el}} \neq \hat{x}_{i}^{\mathrm{el}} \mid x_{\bar{i}}^{\mathrm{el}} \neq \hat{x}_{\bar{i}}^{\mathrm{el}}\right)}_{B} P\left(x_{\bar{i}}^{\mathrm{el}} \neq \hat{x}_{\bar{i}}^{\mathrm{el}}\right) .
\end{aligned}
$$

Because both $x_{i}^{\mathrm{el}}$ and $x_{i}^{\mathrm{el}}$ are equiprobably chosen from the same constellation, their symbol error probability (SER) is the same, that is, $P\left(x_{i}^{\mathrm{el}} \neq \hat{x}_{i}^{\mathrm{el}}\right)=P\left(x_{\bar{i}}^{\mathrm{el}} \neq \hat{x}_{\bar{i}}^{\mathrm{el}}\right)=\mathrm{SER}^{\mathrm{el}}$. Then, (16) becomes

$$
\mathrm{SER}^{\mathrm{el}}=A \cdot\left(1-\mathrm{SER}^{\mathrm{el}}\right)+B \cdot \mathrm{SER}^{\mathrm{el}} .
$$

Here, $A$ can be derived because the ML detection of $x_{i}^{\text {el }}$ is equivalent to the maximal-ratio combining in a single-input multiple-output system since $x_{\bar{i}}^{\mathrm{el}}=\hat{x}_{\bar{i}}^{\mathrm{el}}$. In the appendix, we give the expression of $A$ by the same MGF-based approach. In the process of the ML detection, no operation of hard decision is executed and $\hat{x}_{\bar{i}}^{\text {el }}$ is just a soft symbol. Hence, if $x_{\bar{i}}^{\mathrm{el}} \neq \hat{x}_{\bar{i}}^{\mathrm{el}}$, the probability of decision error cannot be deduced easily so as to hard to derive the expression of B. Here, we utilize the known SPEP to derive the SER. The SPEP also can be expressed as

$$
\begin{aligned}
\operatorname{PEP}^{\mathrm{el}}=\sum_{x_{i}^{\mathrm{e}}, x_{\bar{i}}^{\mathrm{el}} \in C} P\left(x_{i}^{\mathrm{el}}\right) P\left(x_{\bar{i}}^{\mathrm{el}}\right) \\
\cdot\left\{2 P\left(x_{i}^{\mathrm{el}} \neq \hat{x}_{i}^{\mathrm{el}} \mid x_{\bar{i}}^{\mathrm{el}}=\hat{x}_{\bar{i}}^{\mathrm{el}}\right)\left[1-P\left(x_{\bar{i}}^{\mathrm{el}} \neq \hat{x}_{\bar{i}}^{\mathrm{el}}\right)\right]\right. \\
\left.+P\left(x_{i}^{\mathrm{el}} \neq \hat{x}_{i}^{\mathrm{el}} \mid x_{\bar{i}}^{\mathrm{el}} \neq \hat{x}_{\bar{i}}^{\mathrm{el}}\right) P\left(x_{\bar{i}}^{\mathrm{el}} \neq \hat{x}_{\bar{i}}^{\mathrm{el}}\right)\right\} .
\end{aligned}
$$

Utilizing the definition of $A$ and $B$ in (16), (18) can be simplified as

$$
\mathrm{PEP}^{\mathrm{el}}=2 A \cdot\left(1-\mathrm{SER}^{\mathrm{el}}\right)+B \cdot \mathrm{SER}^{\mathrm{el}} .
$$

Then, from (17) and (19), we can get the following closed-form expression of SER:

$$
\mathrm{SER}^{\mathrm{el}}=\frac{\mathrm{PEP}^{\mathrm{el}}-A}{1-A} .
$$

Moreover, the raw BER is scaled to the SER for the square $M_{c^{-}}$ QAM modulation. Therefore, we yield the BER of EL stream as

$$
\mathrm{BER}^{\mathrm{el}}=\frac{\mathrm{SER}^{\mathrm{el}}}{\log _{2}\left(M_{c}\right)} .
$$

In the following, we validate the above deduction by contrasting with the simulation results. As shown in Figure 5, both theoretical and simulated curves of the raw BER for EL of "HM + VR" are overlapped nearly at the medium and high SNR region. This is because our ideal assumption of no error spread in IC is hold when SNR is high enough and what we can calculate is just the upper bound of BER in fact. On the other hand, the argument about diversity gain is also demonstrated by observing the slope of these curves.

3.3. Analysis on Detection Complexity. We discuss the detection complexity for "HM with VR". Here, we apply the QRM [16] detection to (9), which is a popular near-ML lowcomplexity algorithm and explained as follows: first, do QR decomposition to $\widetilde{\mathbf{H}}$, as shown in (22); second, left-multiply $\mathbf{Q}^{H}$ to $\mathbf{y}^{\mathrm{el}}$ of (9); then detect $x_{i}^{\mathrm{el}}$ (i: from 2 to 1 ) in turn because of the up-triangular form of $\mathbf{R}$.

$$
\widetilde{\mathbf{H}}=\beta\left(\begin{array}{cc}
h_{1} & 0 \\
0 & h_{2}
\end{array}\right)\left(\begin{array}{cc}
\cos \varphi & \sin \varphi \\
-\sin \varphi & \cos \varphi
\end{array}\right)=\mathbf{Q R} .
$$

Here, due to $N=2$, QR decomposition can be executed by a simple and ingenious way. Just let

$$
\mathbf{Q}=c\left(\begin{array}{cc}
h_{1} \cos \varphi & h_{2}^{*} \sin \varphi \\
-h_{2} \sin \varphi & h_{1}^{*} \cos \varphi
\end{array}\right),
$$


where $c=\beta^{-1}\left(\left|h_{1}\right|^{2}+\left|h_{2}\right|^{2}\right)^{-1}$. Then

$$
\begin{aligned}
\mathbf{R} & =\mathbf{Q}^{H} \tilde{\mathbf{H}} \\
& =c(\underbrace{\left|h_{1}\right|^{2} \cos ^{2} \varphi+\left|h_{2}\right|^{2} \sin ^{2} \varphi}_{r_{1,1}} \underbrace{\underbrace{h_{1,2} h_{2}}_{r_{2,2}}}_{0} \underbrace{\left.\left|h_{1}\right|^{2}-\left|h_{2}\right|^{2}\right) \sin \varphi \cos \varphi}) .
\end{aligned}
$$

The QR decomposition is completed. This process only needs 13 real multiplications. And the complexity of this preprocessing of detection can nearly be ignored because the channel always keeps constant in a long symbol duration.

In QRM, the heavy complexity lies in the metric computation for each possible symbol candidate. The parameter $M$, which means the number of surviving candidates, can make a tradeoff between complexity and performance. Here, $M=1 \sim 4$ for detecting EL data for QPSK/16 QAM "HM with VR". Table 1 shows the complexity contrast of metric computation between $\mathrm{HM}$ /"HM with CR" and "HM with VR" in detail, where $\mathbf{Q}(i,:)$ denotes the $i$ th row of $\mathbf{Q}$. When $M$ is small, the times of complex multiplication for "HM with VR" is close to that for HM/ "HM with CR". At this time, the performance loss is also acceptable for QPSK/16 QAM "HM with VR".

\section{Performance on Video Quality}

For a fair evaluation of the different transmission schemes in LTE E-MBMS system, the error probability in transmission link is not a proper metric at all. In this paper, we measure the quality of the video with the metric of average peaksignal-to-noise ratio (PSNR) of the video frames, defined as the ratio of the squared maximum pixel value to the mean square error of the reconstructed lossy image at the receiver [17]. In the following, we describe how to estimate the PSNR for the given source rate, and received SNR in transmission link.

4.1. Simulation Method. The derivation of PSNR involves the following three steps.

Step 1 (Get the packet error probability (PER)). For a given information bit rate pair of $\mathrm{BL}$ and EL streams $\left(R^{\mathrm{bl}}, R^{\mathrm{el}}\right)$, we can get their PER pair at the different SNR by Monte Carlo simulation, denoted as $\left(\mathrm{PER}^{\mathrm{bl}}, \mathrm{PER}^{\mathrm{el}}\right)$. It is noted that whether an EL packet is correctly detected or not is on the premise of accurately detecting the BL packet yet.

Step 2 (Estimate the PSNR). We assume that the relation between the bit rate $R$ and the video distortion $D$ (in terms of PSNR) of the transmitted video is known, that is, $D=$ $\mathbb{Q}(R)$, where $\mathbb{Q}(R)$ denotes the operational rate-distortion function of the source coder. The scalable video stream is assumed to truncate at any rate. Hence, the distortion when only $\mathrm{BL}$ is correctly detected will be $D^{\mathrm{bl}}=\mathbb{Q}\left(R^{\mathrm{bl}}\right)$ and the distortion when both BL and EL are correctly detected will be $D^{\text {be }}=\mathbb{Q}\left(R^{\mathrm{bl}}+R^{\mathrm{el}}\right)$. Therefore, if the probability of correctly

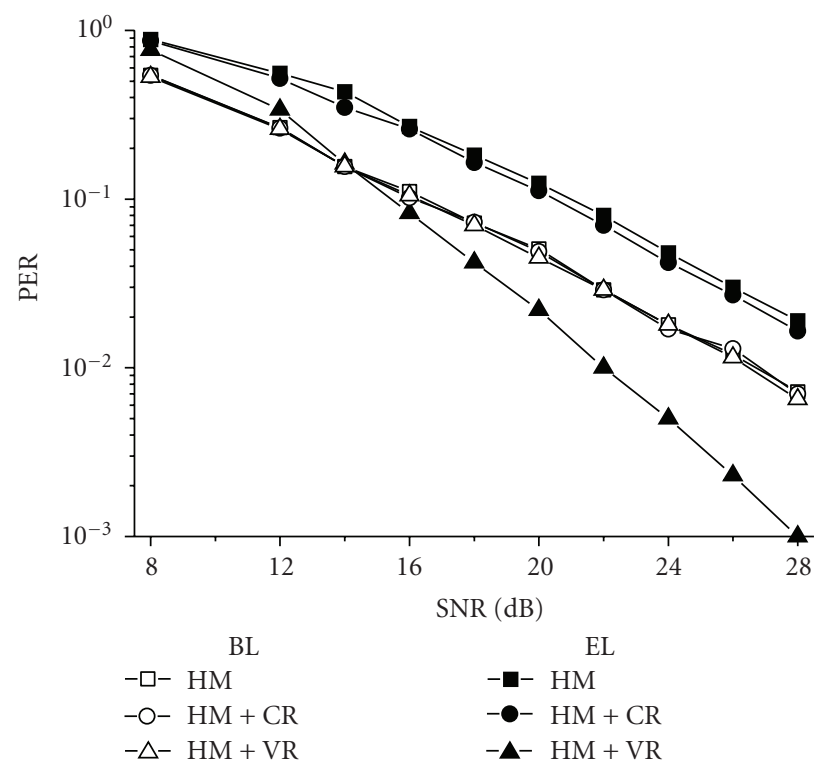

Figure 6: Comparison in link performance.

detecting a packet for each layer is known, the expected PSNR of the received layered video can be found by (referred to $[18]$ )

$$
\begin{aligned}
& D_{\exp }\left(\mathrm{SNR}, R^{\mathrm{bl}}, R^{\mathrm{el}}\right) \\
&=\mathbb{F}_{\mathrm{be}}\left(\mathrm{SNR}, R^{\mathrm{bl}}, R^{\mathrm{el}}\right) \cdot \mathbb{Q}\left(R^{\mathrm{bl}}+R^{\mathrm{el}}\right) \\
&+\left[\mathbb{F}_{\mathrm{b}}\left(\mathrm{SNR}, R^{\mathrm{bl}}\right)-\mathbb{F}_{\mathrm{be}}\left(\mathrm{SNR}, R^{\mathrm{bl}}, R^{\mathrm{el}}\right)\right] \cdot \mathbb{Q}\left(R^{\mathrm{bl}}\right) \\
&=\left[1-\mathrm{PER}^{\mathrm{el}}\right] \cdot \mathbb{Q}\left(R^{\mathrm{bl}}+R^{\mathrm{el}}\right)+\left(1-\mathrm{PER}^{\mathrm{bl}}\right) \\
& \cdot \operatorname{PER}^{\mathrm{el}} \cdot \mathbb{Q}\left(R^{\mathrm{bl}}\right),
\end{aligned}
$$

where $\mathbb{F}_{b}$ is the probability of correctly detecting a packet of $\mathrm{BL}$, which is a function of $\left(\mathrm{SNR}, R^{\mathrm{bl}}\right) ; \mathbb{F}_{\text {be }}$ the probability of detecting both $\mathrm{BL}$ and $\mathrm{EL}$, which is a function of $\left(\mathrm{SNR}, R^{\mathrm{bl}}, R^{\mathrm{el}}\right)$. Then $\left[\mathbb{F}_{\mathrm{b}}-\mathbb{F}_{\mathrm{be}}\right]$ represents probability of detecting only BL but not EL.

4.2. Simulation Results. All the parameters used in our simulations are based on UTRA LTE 3GPP documents [19]. Table 2 shows the respective parameters. Due to the effect of macrodiversity, the MBSFN channel is a 18-path model, in which frequency-selectivity is enough. The power ratio for $\mathrm{HM}$ is constant in the simulation of this section, that is, $\eta=0.7$. Typical data rates for MBMS video streams in CIF resolution is $256 \mathrm{kbps}$. So we set both $R^{\mathrm{bl}}$ and $R^{\mathrm{el}}$ as $256 \mathrm{kbits} / \mathrm{s}$. The used rate-distortion function is referred to [20].

Figure 6 gives the PER of each layer for the three schemes. Their BL performance is similar. However, owning to the higher diversity gain, the EL of "HM with VR" outperforms the other two ELs obviously and is even better than BL at high SNR. That is because the BL of the users at the center 
TABLE 1: Complexity comparison between HM and "HM with VR".

\begin{tabular}{llcccc}
\hline & $\begin{array}{l}\text { Operation for detecting symbols of two } \\
\text { continuous intervals }\end{array}$ & Complex multiplication & Complex addition & Module & Times \\
\hline HM/HM with CR & $\left|y_{i} / h_{i}-\hat{x}_{i}\right| \times 2$ & 1 & 1 & 1 & $2 M_{c}$ \\
& $\left|y_{i}^{\mathrm{bl}} / h_{i}-\hat{x}_{i}^{\mathrm{bl}}\right| \times 2$ & 1 & 1 & 1 & $M_{c} / 4$ \\
HM with VR & $\left|\mathbf{Q}(2,:) \mathbf{y}^{\mathrm{el}}-r_{2,2} \hat{x}_{2}^{\mathrm{el}}\right|$ & 3 & 2 & 1 & $M_{c} / 4$ \\
& $\left|\mathbf{Q}(1,:) \mathbf{y}^{\mathrm{el}}-r_{1,2} \hat{x}_{2}^{\mathrm{el}}-r_{1,1} \hat{x}_{1}^{\mathrm{el}}\right|$ & 3 & 2 & 1 & $M \cdot M_{c} / 4$ \\
\hline
\end{tabular}

TABLE 2: LTE E-MBMS system parameters in simulation.

\begin{tabular}{lc}
\hline Parameters & Values \\
\hline Carrier frequency & $2.0 \mathrm{GHz}$ \\
Bandwidth & $10 \mathrm{MHz}$ \\
Sampling frequency & $15.36 \mathrm{MHz}$ \\
Subcarrier space & $15 \mathrm{KHz}$ \\
Used resource block & 2 \\
Number of antennas & $1 \times 1$ \\
Simulation unit & 1 subframe \\
OFDM symbols & 12 symbols/subframe \\
FFT size & 1024 \\
CP size & 256 \\
Channel model & MBSFN Propagation Channel [21] \\
Velocity & $2.7 \mathrm{~km} / \mathrm{h}$ \\
Modulation & HM, HM + CR, HM + VR (QPSK/16 QAM) \\
Code rate & 0.5 \\
\hline
\end{tabular}

is protected enough. At this time, it is not necessary to stress that BL should get more protection than EL, although this condition is significant when SNR is relatively low. On the other hand, the EL of "HM with CR" only has a slight advantage over that of HM due to the limited increase of MED. Figure 7 shows the video quality comparison. Let PSNR $=32 \mathrm{~dB}$ as the quality benchmark, our proposed "HM with VR" can save $1.5 \mathrm{~dB}$ in received SNR comparing with the regular $\mathrm{HM}$ and $1.1 \mathrm{~dB}$ comparing with "HM with CR". The saving SNR means the lower transmit power requirement or the larger coverage.

\section{Performance on Coverage}

5.1. Simulation Method. To illuminate the benefit on coverage more clearly, the power ratio is adjusted to evaluate the supported distance between the BS and the UE at the cell edge with the PSNR benchmark.

Step 1 (Determine the required SNR). Firstly, adjust the power ratio $\eta$ from 0.6 to 1 . For each certain $\eta$, we can obtain a group of curves of expected PSNR versus received SNR similar to that in Figure 7. According to the benchmark, we can record the required SNR values for the three schemes, respectively, from the curves.

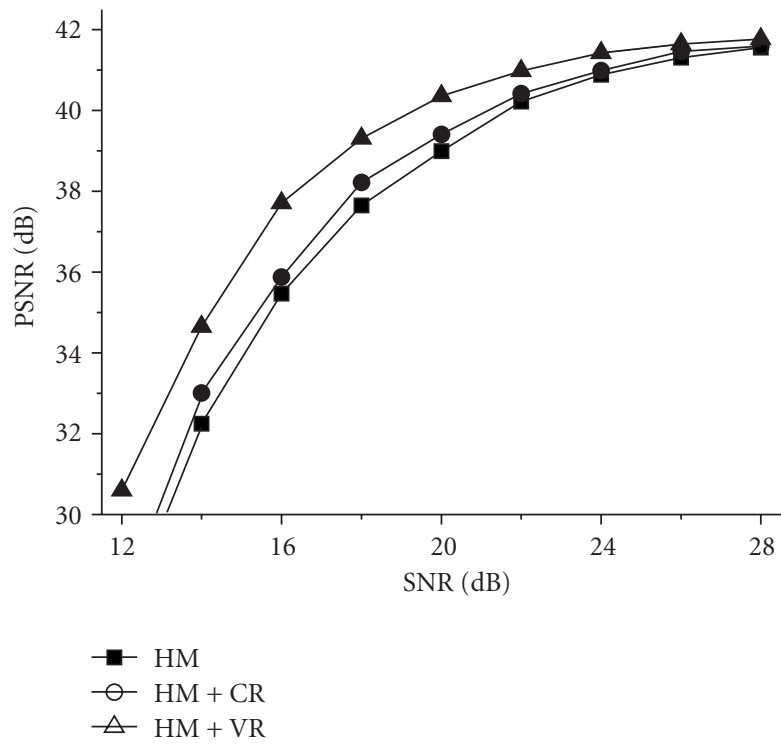

FIgURE 7: Comparison in video quality.

Step 2 (Compute the distance). Rooting in the path loss formula, the relation between the distance $d$ (i.e., the cell radius, in meter) and average received SNR (in $\mathrm{dB}$ ) is

$$
\mathrm{SNR}=\mathrm{SNR}_{t}-10 \alpha \log _{10} d,
$$

where $\mathrm{SNR}_{t}$ is the transmit SNR; $\alpha$ is the path loss constant and is set as 3.76. We consider a coverage range up to about 300 meters because the distance between two BS is 500 meters. $\mathrm{SNR}_{t}$ is chosen so that the received SNR is $5 \mathrm{~dB}$ at the cell edge. Therefore, according the recorded SNR values in Step 1, we can derive the distance for any HM scheme with any power ratio.

5.2. Simulation Results. With the different power allocation to each layer, Figure 8 shows the corresponding coverage distances for the three schemes. Firstly, the coverage is increasing with $\eta$ because the allocated power to $\mathrm{BL}$ is to guarantee the reception of users at the edge. However, if the power of BL is too large, EL stream cannot be decoded correctly so that PSNR is deteriorated. For guaranteeing the PSNR benchmark, the coverage distance will be shortened. Hence, the distance is optimal for these schemes when $\eta$ is about 0.85 . We discount the distance to coverage area. Then, our proposed "HM with VR" can extend the coverage by $10 \%$ and 7\%, respectively, relative to HM and "HM with CR". 


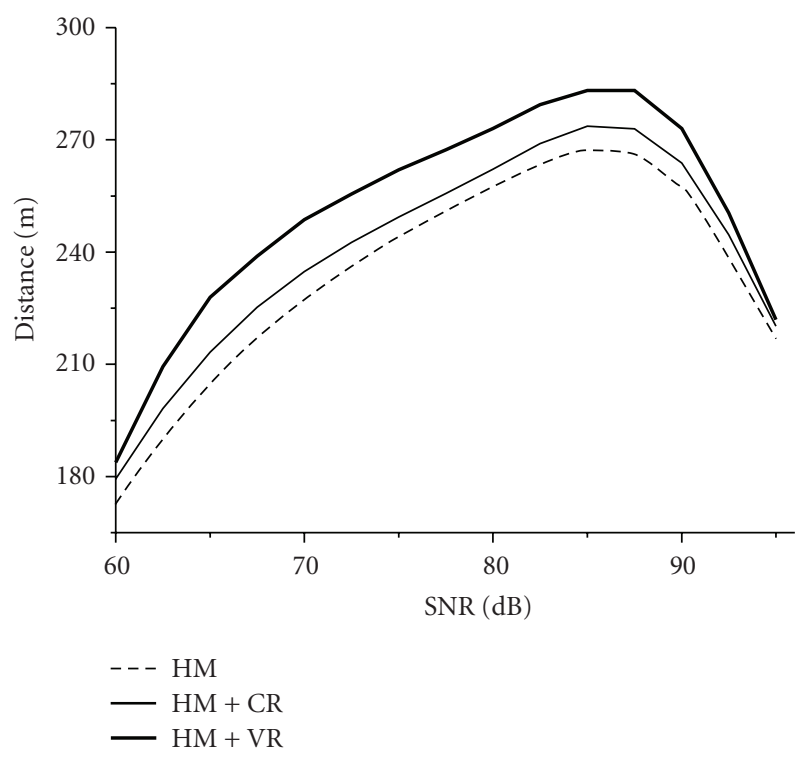

Figure 8: Comparison in coverage.

\section{Conclusion}

In this paper, we focus on the application of $\mathrm{HM}$ for EMBMS in LTE system. After introducing the regular HM and an enhanced HM with CR, we proposed an HM with $\mathrm{VR}$ and its corresponding detection algorithm to improve the performance of less-protected enhancement-layer data stream, in which the idea stems from the concept of signal space diversity in single antenna system. With the suggestion of the dimension of rotated vector $N=2$, we proved that the EL can earn the full diversity by both SPEP derivation and BER simulation. At the same time, the analysis of detection complexity when $N=2$ indicates that "HM with VR" will not incur the heavy complexity. We evaluate the proposed scheme in a more integrative metric, not the common error probability, but the video distortion measured by PSNR. The simulation results showed that the proposed "HM with VR" can save $1.5 \mathrm{~dB}$ compared with HM, $1.1 \mathrm{~dB}$ with "HM with CR" on the required SNR to reach the video quality benchmark. Besides, the simulation results about coverage showed that the extended coverage area ratios are $10 \%$ and $7 \%$, respectively.

\section{Appendix}

In the appendix, we deduce the expression of $P\left(x_{i}^{\mathrm{el}} \neq \hat{x}_{i}^{\mathrm{el}} \mid\right.$ $\left.x_{\bar{i}}^{\mathrm{el}}=\hat{x}_{\bar{i}}^{\mathrm{el}}\right)$ in $(16)$.

Due to $N=2$ and $x_{\bar{i}}^{\mathrm{el}}=\hat{x}_{\bar{i}}^{\mathrm{el}}$, the ML detection of $x_{i}^{\mathrm{el}}$ is equivalent to the maximal-ratio combining (MRC) in a single-input multiple-output system. Thus, the post-SNR of $x_{i}^{\mathrm{el}}$ after MRC is

$$
\gamma=\sum_{j=1}^{2} \frac{\left|[\tilde{\mathbf{H}}]_{i, j}\right|^{2}\left|\delta_{i}\right|^{2}}{\sigma_{n}^{2}}=\frac{\beta\left(\left|h_{1}\right|^{2}+\left|h_{2}\right|^{2}\right)\left|\delta_{i}\right|^{2}}{\sigma_{n}^{2}}
$$

where $[\tilde{\mathbf{H}}]_{i, j}$ denotes the element at $i$ th row and $j$ th column. Then, the conditional SER of EL is given by

$$
\begin{aligned}
P\left(x_{i}^{\mathrm{el}}\right. & \left.\neq \hat{x}_{i}^{\mathrm{el}} \mid x_{\bar{i}}^{\mathrm{el}}=\hat{x}_{\bar{i}}^{\mathrm{el}}, \tilde{\mathbf{H}}\right) \\
& =Q(\sqrt{\gamma})=Q\left(\sqrt{\frac{\beta\left(\left|h_{1}\right|^{2}+\left|h_{2}\right|^{2}\right)\left|\delta_{i}\right|^{2}}{\sigma_{n}^{2}}}\right) .
\end{aligned}
$$

By the same MGF-based approach, we can yield the analytic expression of $P\left(x_{i}^{\mathrm{el}} \neq \hat{x}_{i}^{\mathrm{el}} \mid x_{\bar{i}}^{\mathrm{el}}=\hat{x}_{\bar{i}}^{\mathrm{el}}\right)$ after taking the expectation of channel element:

$$
P\left(x_{i}^{\mathrm{el}} \neq \hat{x}_{i}^{\mathrm{el}} \mid x_{\bar{i}}^{\mathrm{el}}=\hat{x}_{\bar{i}}^{\mathrm{el}}\right)=\frac{1}{\pi} \int_{0}^{\pi / 2}\left(\frac{\beta^{2}\left|\delta_{i}\right|^{2}}{2 \sigma_{n}^{2} \sin ^{2} \theta}\right)^{-2} d \theta=\frac{3 \sigma_{n}^{4}}{4 \beta^{4}\left|\delta_{i}\right|^{4}} .
$$

\section{Acknowledgment}

This work was supported by National Hi-Tech R\&D 863 Program of China under Grant no. 2009AA01Z244.

\section{References}

[1] M. Effros, "Universal multiresolution source codes," IEEE Transactions on Information Theory, vol. 47, no. 6, pp. 21132129, 2001.

[2] S. Borade, B. Nakiboğlu, and L. Zheng, "Some fundamental limits of unequal error protection," in Proceedings of the IEEE International Symposium on Information Theory (ISIT '08), pp. 2222-2226, July 2008.

[3] G. Li, Z. Zhang, and Z. Lu, "Adaptive hierarchical modulation over correlated MIMO fading channels," in Proceedings of the 9th International Conference on Signal Processing (ICSP '08), pp. 1920-1925, October 2008.

[4] S. A. Ramprashad and C.-E. W. Sundberg, "Hierarchical QAM BICM MIMO systems with iterative decoding and applications to media broadcast," in Proceedings of the 9th IEEE International Symposium on Wireless, Mobile and Multimedia Networks (WoWMoM '08), pp. 1-9, June 2008.

[5] N. Souto, A. Correia, R. Dinis, J. C. Silva, and L. Abreu, "Multiresolution MBMS transmissions for MIMO UTRA LTE systems," in Proceedings of IEEE International Symposium on Broadband Multimedia Systems and Broadcasting (BMSB '08), pp. 1-6, April 2008.

[6] A. Correia, N. Souto, A. Soares, R. Dinis, and J. Silva, "Multiresolution with hierarchical modulations for long term evolution of UMTS," EURASIP Journal on Wireless Communications and Networking, vol. 2009, Article ID 240140, pp. 1-12, 2009.

[7] D. Pradas, A. Bouabdallah, J. Lacan, M. A. Vázquez Castro, and M. Bousquet, "Cross-layer optimization of unequal protected layered video over hierarchical modulation," in Proceedings of the IEEE Global Telecommunications Conference (GLOBECOM '09), pp. 1-6, 2009.

[8] S. S. Arslan, P. C. Cosman, and L. B. Milstein, "Progressive source transmissions using joint source-channel coding and hierarchical modulation in packetized networks," in Proceedings of the IEEE Global Telecommunications Conference (GLOBECOM '09), pp. 1-6, 2009. 
[9] S. Wang, S. Kwon, and B. K. Yi, "On enhancing hierarchical modulation," in Proceedings of the IEEE International Symposium on Broadband Multimedia Systems and Broadcasting (BMSB '08), pp. 1-6, April 2008.

[10] S. M. S. Sadough and P. Duhamel, "On the interaction between channel coding and hierarchical modulation," in Proceedings of the IEEE International Conference on Communications (ICC '09), pp. 1-5, June 2009.

[11] J. Boutros and E. Viterbo, "Signal space diversity: a power-and bandwidth-efficient diversity technique for the rayleigh fading channel," IEEE Transactions on Information Theory, vol. 44, no. 4, pp. 1453-1467, 1998.

[12] T. M. Cover and J. A. Thomas, Elements of Information Theory, 2nd edition, 2006.

[13] P. K. Vitthaladevuni and M.-S. Alouini, "A recursive algorithm for the exact BER computation of generalized hierarchical QAM constellations," IEEE Transactions on Information Theory, vol. 49, no. 1, pp. 297-307, 2003.

[14] M. K. Simon and M. S. Alouini, Digital Communication over Fading Channels, John Wiley \& Sons, New York, NY, USA, 2005.

[15] J. W. Craig, "A new, simple and exact result for calculating the probability of error for two-dimensional signal constellations," in Proceedings of the IEEE Military Communications Conference (MILCOM '91), pp. 2551-2555, November 1991.

[16] K. Higuchi, H. Kawai, N. Maeda, and M. Sawahashi, "Adaptive selection of surviving symbol replica candidates based on maximum reliability in QRM-MLD for OFCDM MIMO multiplexing," in Proceedings of the IEEE Global Telecommunications Conference (GLOBECOM '04), pp. 2480-2486, December 2004.

[17] Q. Huynh-Thu and M. Ghanbari, "Scope of validity of PSNR in image/video quality assessment," Electronics Letters, vol. 44, no. 13, pp. 800-801, 2008.

[18] Ç. Bilen, E. Erkip, and Y. Wang, "Layered video multicast using diversity embedded space time codes," in Proceedings of the 32nd International Conference on Sarnoff Symposium (SARNOFF'09), pp. 1-5, April 2009.

[19] 3GPP TS 36.212 v8.7.0 "E-UTRA Physical channels and modulation", 2009.

[20] V. Vukadinović and J. Huschke, "Statistical multiplexing gains of H.264/AVC video in E-MBMS," in Proceedings of the 3rd International Symposium on Wireless Pervasive Computing (ISWPC '08), pp. 468-474, May 2008.

[21] R4-094198, "LTE MBSFN Channel Model," Ericsson, 3GPP TSG-RAN4 \#53 Jeju, Korea, 2009. 

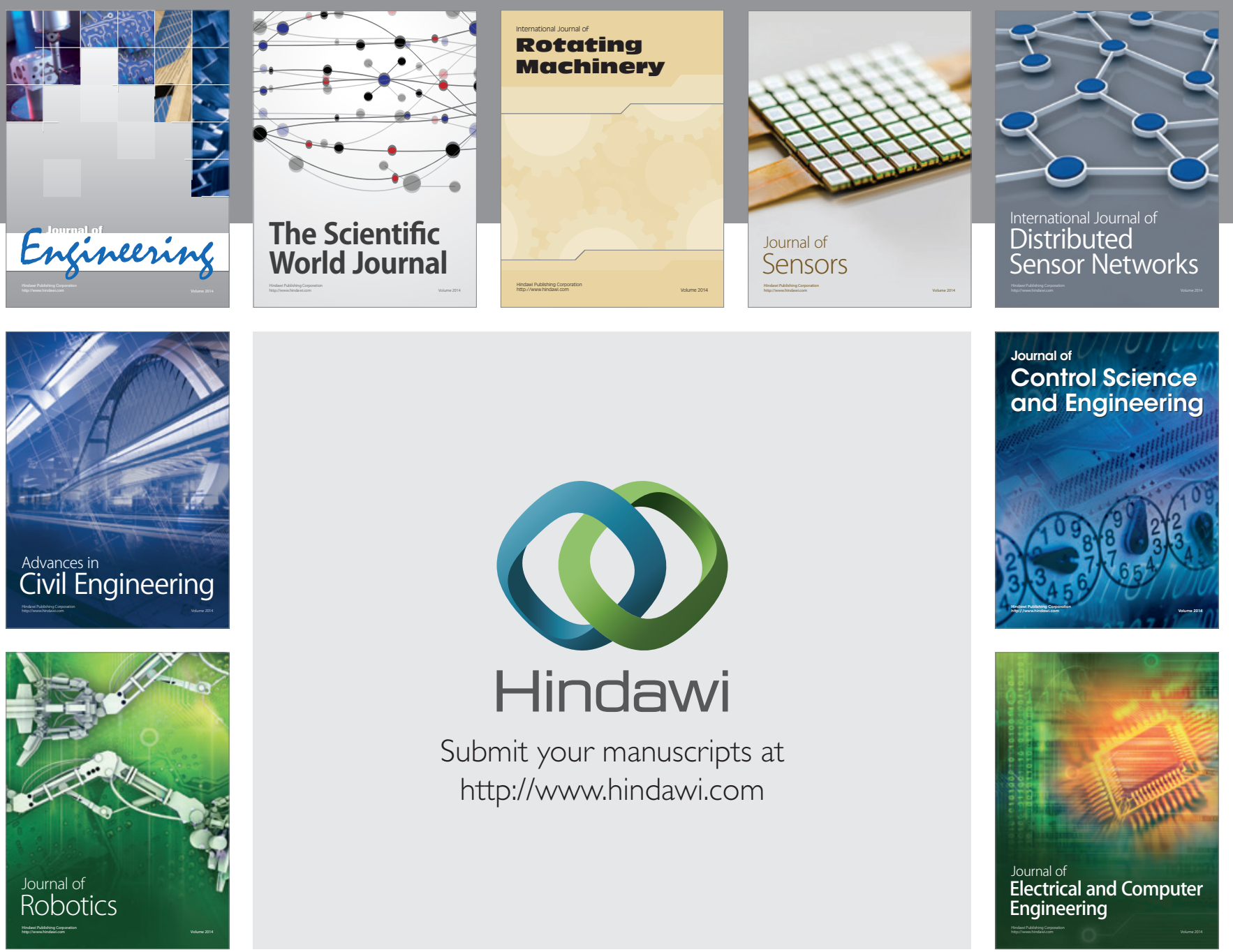

Submit your manuscripts at

http://www.hindawi.com
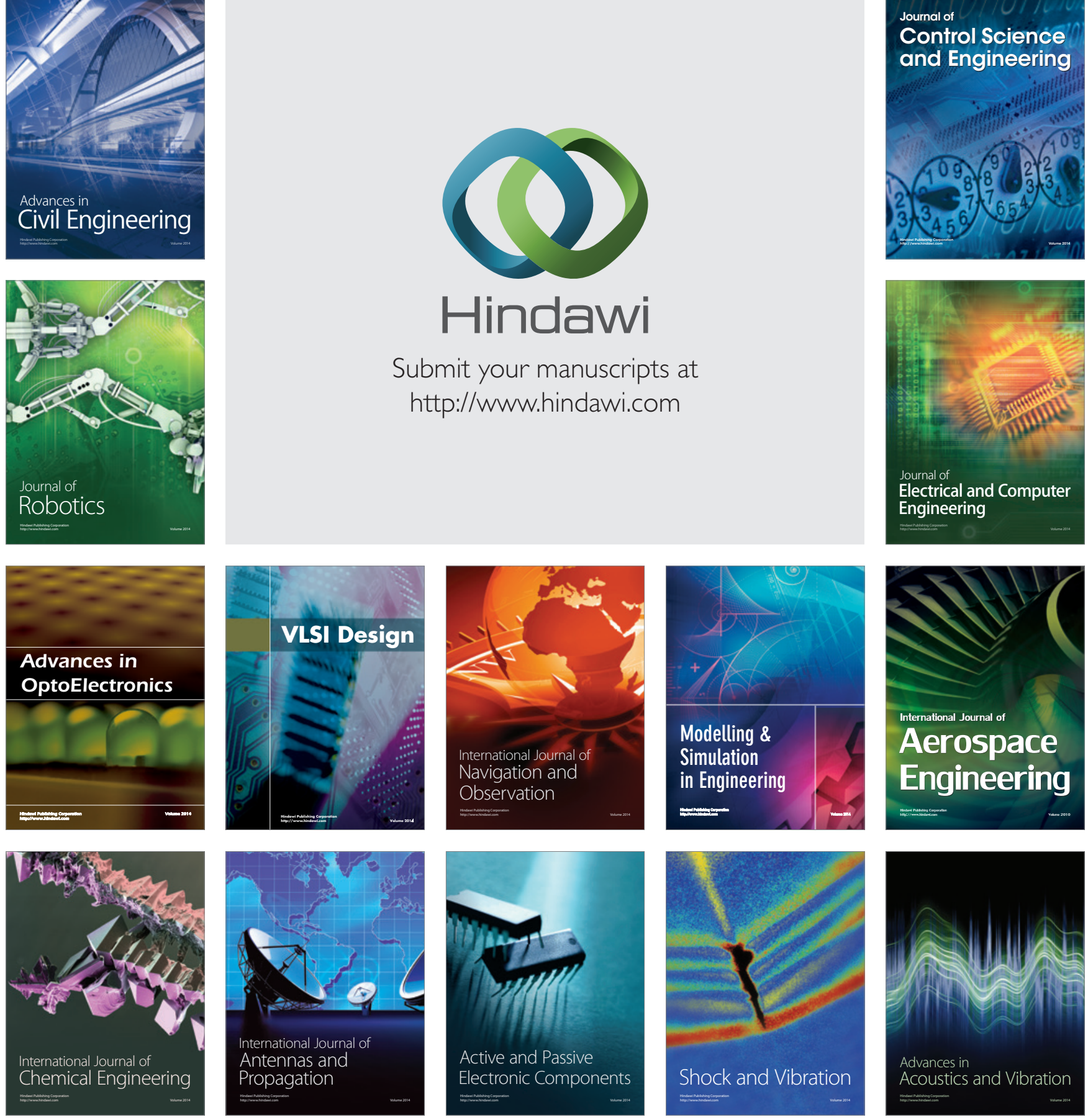